\title{
Hypertensive disorders of pregnancy, respiratory outcomes and atopy in childhood
}

\author{
Seif 0. Shaheen ${ }^{1}$, Corrie Macdonald-Wallis ${ }^{2,3}$, Debbie A. Lawlor ${ }^{2,3}$ \\ and A. John Henderson ${ }^{2}$
}

Affiliations: ${ }^{1}$ Centre for Primary Care and Public Health, Barts and The London School of Medicine and Dentistry, London, UK. ${ }^{2}$ School of Social and Community Medicine, University of Bristol, Bristol, UK. ${ }^{3} \mathrm{MRC}$ Integrative Epidemiology Unit, University of Bristol, Bristol, UK.

Correspondence: Seif 0 . Shaheen, Centre for Primary Care and Public Health, Blizard Institute, Barts and The London School of Medicine and Dentistry, 58 Turner Street, London, E1 2AB, UK.

E-mail: s.shaheendamul.ac.uk

ABSTRACT Few epidemiological studies have investigated the role of hypertensive disorders of pregnancy in the aetiology of childhood respiratory and atopic outcomes.

In the Avon Longitudinal Study of Parents and Children we examined associations of maternal gestational hypertension, hypertension before pregnancy and pre-eclampsia with wheezing at 18 months, wheezing and asthma at 7 years and lung function at 8-9 years, after controlling for potential confounders ( $\mathrm{n}=5322-8734$, depending on outcome).

Gestational hypertension was not associated with any of the outcomes. There was weak evidence for a positive association between pre-eclampsia and early wheezing (adjusted OR 1.31, 95\% CI 0.94-1.82, compared to normotensive pregnancies) and for negative associations between pre-eclampsia and forced expiratory volume in $1 \mathrm{~s}$ (adjusted mean difference in SD score $-0.14,95 \% \mathrm{CI}-0.33-0.06$ ) and maximal mid-expiratory flow $(-0.15,95 \%$ CI $-0.34-0.04)$. Hypertension before pregnancy was positively associated with wheezing (OR 1.63, 95\% CI 1.16-2.31) and asthma (OR 1.34, 95\% CI 1.00-1.79).

Gestational hypertension is unlikely to be a risk factor for childhood respiratory disorders; hypertension before pregnancy may be a risk factor for childhood wheezing and asthma, but this finding needs replication. Larger studies are needed to confirm whether pre-eclampsia is associated with impaired childhood lung function.

@ERSpublications

Gestational hypertension is unlikely to be a risk factor for childhood respiratory disorders http://ow.ly/StqFo

\footnotetext{
This article has supplementary material available from erj.ersjournals.com

Received: Sept 022014 | Accepted after revision: Aug 272015 | First published online: Nov 052015

Support statement: The UK Medical Research Council (MRC), the Wellcome Trust (grant number: 092731) and the University of Bristol currently provide core support for the Avon Longitudinal Study of Parents and Children (ALSPAC). C. Macdonald-Wallis and D.A. Lawlor work in a unit that receives funding from the MRC (grant number MC_UU_12013/5). C. Macdonald-Wallis is funded by a UK MRC research fellowship (grant number MR/J011932/1). Funding information for this article has been deposited with FundRef.
}

Conflict of interest: Disclosures can be found alongside the online version of this article at erj.ersjournals.com

Copyright CERS 2016 


\section{Introduction}

There is a growing body of epidemiological evidence implicating the prenatal environment in the aetiology of childhood respiratory disorders such as asthma [1]. Risk factors that deserve investigation include the hypertensive disorders of pregnancy (HDP), namely gestational hypertension, pre-eclampsia and existing (chronic) hypertension. Hypertension in pregnancy has been associated with impaired infant lung function in an Australian cohort [2]. There is a paucity of data on the relation between HDP and lung function later in childhood, although one small Norwegian historical cohort study found no association between pre-eclampsia and childhood lung function [3]. A recent pooled analysis of 14 European birth cohorts reported positive associations between pre-eclampsia and ever/recurrent wheezing at 12-24 months of age [4]. Maternal hypertension in pregnancy or pre-eclampsia, analysed in combination, were positively associated with an increased risk of early, late onset and persistent wheezing in the first 6-7 years of life in a large Italian cross-sectional study, although information about HDP was based on maternal recall [5]. In contrast, a Norwegian birth cohort found no association between pregnancy complications (hypertension, pre-eclampsia or hyperemesis combined) and bronchial obstruction before 2 years of age or asthma at 4 years [6]. Two other Norwegian studies found no relation between pre-eclampsia and asthma in the offspring $[3,7]$, and a British birth cohort study found no association between maternal hypertension in pregnancy and offspring asthma [8]. A potential limitation of studies to date is that few have distinguished associations with gestational hypertension, hypertension before pregnancy and pre-eclampsia separately. While gestational hypertension and pre-eclampsia are currently thought to represent a spectrum of severity of a single condition, with pre-eclampsia at the severe end [9], guidelines for clinical management are different for each of the hypertensive disorders [10], and thus it would seem sensible to analyse the disorders separately if possible.

One small Finnish study $(n=378)$ found that females who experienced either pre-eclampsia or placental abruption in pregnancy had offspring with an increased risk of severe atopy during adolescence, an association which was limited to males [11]. A larger Norwegian study reported that severe (but not mild/ moderate) pre-eclampsia was also associated with a high level of allergic sensitisation [3].

In the Avon Longitudinal Study of Parents and Children (ALSPAC) birth cohort we have investigated whether HDP are associated with an increased risk of early and later wheezing, asthma, impaired lung function and atopy in childhood.

\section{Methods}

Participants

ALSPAC is a population-based birth cohort that recruited 14541 predominantly white pregnant females resident in Avon, UK with expected dates of delivery April 1, 1991 to December 31, 1992. Of these pregnancies there were 14676 fetuses, resulting in 14062 live births and 13978 singleton or twin children who were alive at 1 year of age. Of these, 13758 had maternal obstetric information abstracted from medical records. The cohort has been followed since birth with annual questionnaires and, since the age of 7 years, with objective measures in annual research clinics. The study protocol has been described previously $[12,13]$ and further information can be found at www.alspac.bris.ac.uk. The study website contains details of all the data that are available through a fully searchable data dictionary: www.bris.ac.uk/ alspac/researchers/data-access/data-dictionary. Ethics approval for all aspects of data collection was obtained from the ALSPAC ethics and law committee (institutional review board 00003312) and the local National Health Service research ethics committees.

\section{Hypertensive disorders of pregnancy}

All blood pressure and proteinuria measurements, which were taken as part of routine antenatal care by midwives or obstetricians, were abstracted from obstetric records by six trained research midwives. There was no between-midwife variation in mean values of the data abstracted and error rates were consistently $<1 \%$ in repeated data entry checks. These data were used to derive three HDP using the widely used International Society for the Study of Hypertension in Pregnancy criteria [14], which are used clinically in the UK. Gestational hypertension was defined as systolic blood pressure $\geqslant 140 \mathrm{mmHg}$ and/or diastolic blood pressure $\geqslant 90 \mathrm{mmHg}$ on two occasions after 20 weeks' gestation in a mother who did not report having hypertension prior to pregnancy. Pre-eclampsia was defined by the same blood pressure thresholds with proteinuria of $\geqslant 1+$ on urine dipstick testing occurring at the same time as the elevated blood pressure. Subjects who responded to a questionnaire administered during pregnancy that they had previously had hypertension outside of pregnancy were classified as having hypertension before pregnancy.

\section{Outcomes}

When the children were 18 months old, their mothers were asked whether the child had wheezed in the previous 12 months and whether their child had "wheezing with whistling on the chest when (s)he 
breathed". When the children were 7.5 years old, mothers were asked "Has your child had any of the following in the past 12 months: wheezing; asthma?" Children were defined as having current doctor-diagnosed asthma at 7.5 years (primary outcome of interest) if mothers responded positively to the question "Has a doctor ever actually said that your study child has asthma?" and positively to one or both of the questions on wheezing and asthma in the past 12 months.

Lung function was measured by spirometry (Vitalograph 2120; Vitalograph, Maids Moreton, UK) at age 8.5 years after withholding short-acting bronchodilators for $\geqslant 6 \mathrm{~h}$ and long-acting bronchodilators and theophyllines for $\geqslant 24 \mathrm{~h}$. The best of three reproducible flow-volume curves was used to measure forced expiratory volume in $1 \mathrm{~s}$ (FEV1), forced vital capacity (FVC) and forced expiratory flow at 25-75\% of FVC (FEF25-75\%). Lung function measurements were transformed to age-, height- and sex-adjusted standard deviation units [15]. The tests adhered to American Thoracic Society (ATS) criteria for standardisation and reproducibility of flow-volume measurement [16], with the exception of ATS recommendations for duration of expiration [17]; as many children did not fulfil the end-of-test criteria of forced expiratory time $>6 \mathrm{~s}$, a minimal volume change over the final $1 \mathrm{~s}$ was used.

Atopy at 7 years was defined as a positive reaction (any detectable weal) to Dermatophagoides pteronyssinus, cat or grass (after subtracting positive saline reactions from histamine and allergen weals, and excluding children who were unreactive to $1 \%$ histamine). Atopy defined in this way identified $96 \%$ of children sensitised to 26 other allergens in this cohort [18].

\section{Maternal covariables}

Maternal age at delivery was abstracted from obstetric records. Maternal asthma, pre-pregnancy weight and height, parity, smoking status, highest educational qualification and ethnicity were obtained from questionnaires administered during pregnancy. Maternal asthma was defined as a positive response to having had asthma either recently or in the past. Pre-pregnancy body mass index (BMI) was calculated as weight/ height ${ }^{2}$ and classed according to World Health Organization definitions of underweight $\left(<18.5 \mathrm{~kg} \cdot \mathrm{m}^{-2}\right)$, normal weight $\left(18.5-24.9 \mathrm{~kg} \cdot \mathrm{m}^{-2}\right)$, overweight $\left(25.0-29.9 \mathrm{~kg} \cdot \mathrm{m}^{-2}\right)$ and obese $\left(\geqslant 30.0 \mathrm{~kg} \cdot \mathrm{m}^{-2}\right)$. Smoking status was categorised as the maximum maternal exposure during pregnancy (never, passive smoking only, 1-9 cigarettes per day, $10-19$ cigarettes per day and $\geqslant 20$ cigarettes per day). Maternal ethnicity was categorised as white or nonwhite.

\section{Statistical analyses}

We compared the distributions of child and maternal variables across HDP categories using f-statistics for differences in the means of continuous variables and Chi-squared tests for differences in the categorical variables.

We used logistic regression to relate maternal HDP to binary offspring outcomes: wheezing, asthma and atopy. We present odds ratios for these outcomes comparing children whose mothers had gestational hypertension, pre-eclampsia and hypertension before pregnancy (separately) with children of normotensive pregnancies (the reference category). Linear regression was used to relate maternal HDP to the continuous lung function outcomes FEV1, FVC and FEF25-75\%. Mean differences in the age, sex and height-standardised SD scores [15] for these outcomes associated with maternal gestational hypertension and pre-eclampsia compared with normotensive pregnancies are presented.

For both the logistic and linear regression analyses, four stages of adjustment were used. We selected potential confounding factors which are known (from existing literature) to be associated with HDP and one or more of our outcomes of interest. In model 1 we adjusted for offspring sex only. In model 2 we adjusted additionally for the potential maternal confounders of maternal asthma, pre-pregnancy BMI, age, parity, highest educational qualification, twin pregnancy and ethnicity. In model 3 we adjusted additionally for maternal smoking during pregnancy. This was included in a separate stage of adjustment, since maternal smoking during pregnancy has been shown to be negatively associated with HDP [19] but also associated with increased risk of asthma in the child, meaning that it had the potential to negatively confound and mask, rather than inflate, any effect of maternal HDP on these outcomes. By way of subsidiary analyses, we additionally adjusted for gestational age at delivery and birthweight to explore whether prematurity or impaired fetal growth might mediate associations between HDP and childhood outcomes. We have made some key assumptions which are necessary for mediation analysis, namely that there is no interaction between the exposures and mediators [20] and no unmeasured confounding of the mediator-outcome associations [21]. Online supplementary figure E1 shows a directed acyclic graph to illustrate potential confounders and mediators of the associations between HDP and childhood outcomes. All statistical analyses were completed using Stata (version 12.1; StataCorp LP, College Station, TX, USA). 


\section{Results}

Figure 1 shows participant flow in the ALSPAC birth cohort, and numbers of mother/child pairs with complete data according to childhood outcomes. Characteristics of mothers and offspring who were included in the analyses of each childhood outcome and who were excluded from all analyses because of incomplete data are shown in online supplementary table E1. Mothers with incomplete data were more likely to be smokers, less well educated, multiparous, younger and nonwhite than mothers who were included. Excluded offspring had lower lung function and lower birthweight and a higher prevalence of early and later wheezing and asthma than those who were included, although differences were small.

Table 1 shows the distribution of maternal and offspring characteristics, including respiratory and atopic outcomes, according to maternal HDP. The prevalences of gestational hypertension, pre-eclampsia and hypertension before pregnancy were $14 \%, 2 \%$ and $4 \%$, respectively. Children of mothers who had pre-eclampsia had lower FEF25-75\% and lower gestational age and birthweight than children of other mothers. The prevalence of wheeze at 7 years was higher in children of mothers who had hypertension before pregnancy, but the prevalence of early wheezing and later asthma and atopy did not differ according to HDP. Mothers who had gestational hypertension and pre-eclampsia were more likely than normotensive mothers to be carrying twin pregnancies, and mothers with HDP were more likely to be nulliparous and to have higher pre-pregnancy BMI, and less likely to have actively smoked during pregnancy. Mothers with gestational hypertension and hypertension before pregnancy were more likely than normotensive mothers to have asthma.

After controlling for confounders (model 3), gestational hypertension was not associated with wheezing at 18 months or 7 years; however, there was weak evidence for a positive association between pre-eclampsia and early wheezing and stronger evidence for a positive association between hypertension before pregnancy and wheezing at 7 years (table 2 and fig. 2). After controlling for confounders (model 3), there was evidence for a positive association between hypertension before pregnancy and asthma at 7 years, but neither gestational hypertension nor pre-eclampsia was associated with asthma; none of the HDP were associated with atopy at 7 years (tables 3 and 4 and fig. 2).

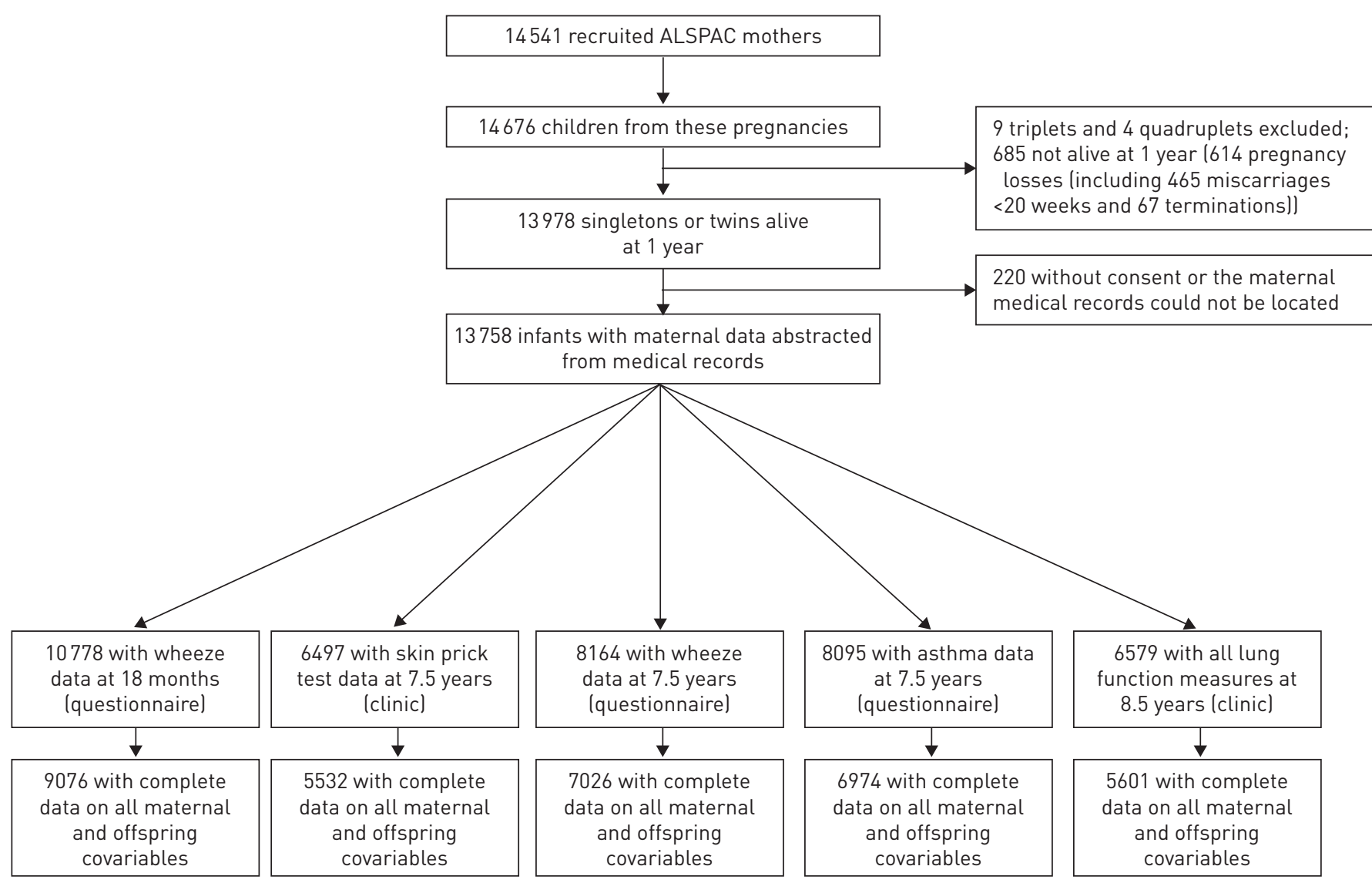

FIGURE 1 Participant flow. ALSPAC: Avon Longitudinal Study of Parents and Children. 
TABLE 1 Characteristics of mothers and offspring who had information on at least one set of outcome variables lwheeze, asthma, lung function or atopyl by maternal hypertensive disorders of pregnancy

\section{Total subjects}

Normotensive
Gestational
hypertension
Pre-eclampsia

$\begin{array}{cc}\begin{array}{c}\text { Hypertension } \\ \text { before pregnancy }\end{array} & \begin{array}{c}p \text {-value } \\ \text { for } \\ \text { difference }\end{array}\end{array}$

420

$71.3(n=285)$
$28.8(n=115)$
$84.1(n=260)$
$15.9(n=49)$

$27.7(\mathrm{n}=2378)$

$74.0(n=1154)$

$26.0(n=405)$

70.9 ( $n=158)$

$29.1(n=65)$

$88.4(n=1020)$

$11.6(n=134)$

$88.9(n=144)$

$10.4(n=678)$

Female

Multiple pregnancy

Singleton

Twin

Maternal ethnicity

White

Nonwhite

Maternal pre-pregnancy

BMI $\mathrm{kg} \cdot \mathrm{m}^{-2}$

Maternal age years

Parity

Nulliparous

Multiparous

Maternal smoking

in pregnancy

None $\quad 4902$

Passive $\quad 3711$

1-9 cigarettes per day 987

10-19 cigarettes per day $\quad 1214$

$\geqslant 20$ cigarettes per day 553

Maternal education

Certificate of Secondary 3003

Education/vocational

Ordinary level

Advanced level

Degree

Maternal asthma

No 9797

Yes $\quad 1256$

Gestational age weeks $\rrbracket$

Birthweight $g$ $79.9(n=5185)$

$20.1(n=1301)$

$-0.01 \pm 0.99(n=5238)$

$-0.01 \pm 0.99(n=5323)$

$0.00 \pm 1.01(n=5323)$

6676

5158

1339

5980

5621 $79.5(n=4114)$

20.5 ( $n=1063)$

$51.0(n=4729)$

$49.0(n=4539)$

97.7 ( $n=9058$ )

$2.3(n=210)$

$97.6(n=8562)$

$2.4(\mathrm{n}=211)$

$22.50 \pm 3.33(n=8271)$

$28.50 \pm 4.74(n=9268)$

$41.9(n=3724)$

$58.1(n=5160)$

$42.8(n=3893)$

$31.7(n=2884)$

$9.0(n=819)$

$11.3(n=1027)$

$5.1(n=467)$

$27.6(n=2432)$

$34.6(n=3048)$

$24.0(n=2119)$

$13.8(n=1212)$

$89.1(n=7851)$

$10.9(n=963)$

40 (39-41) ( $n=9268)$

$3421 \pm 531(n=9150)$

\section{$79.1(n=902)$}

20.9 ( $n=238)$

$0.03 \pm 1.03(n=957)$

$0.06 \pm 1.03(n=964)$

$-0.03 \pm 0.99(n=964)$

$79.1(n=743)$

$20.9(n=196)$

$53.7(\mathrm{n}=899)$

$46.3(n=776)$

$95.3(n=1597)$

$4.7(n=78)$

$98.8(n=1569)$

$1.2(n=19)$

$24.67 \pm 4.69(n=1450)$

$28.31 \pm 4.86(n=1675)$

$57.0(n=910)$

$43.0(n=686)$

$43.5(n=710)$

$36.9(n=603)$

$7.6(n=124)$

$8.1(n=132)$

$3.9(n=64)$

$25.8(n=411)$

$37.7(n=600)$

$22.2(n=354)$

$14.3(n=228)$

$87.0(n=1387)$

$13.0(n=208)$

11460
40 (39-41) ( $n=1675)$

$3420 \pm 587$ ( $n=1658$ ) $11.1(n=18)$

$74.5(n=120)$

$25.5(\mathrm{n}=41)$

$-0.09 \pm 1.01(n=138)$

$-0.01 \pm 1.01(n=138)$

$-0.18 \pm 0.91(n=138)$

$81.1(n=107)$

$18.9(n=25)$

$52.5(n=125)$

47.5 ( $n=113$ )

$94.1(n=224)$

$5.9(n=14)$

$97.3(n=214)$

$2.7(n=6)$

$24.64 \pm 5.28(n=208)$

$27.82 \pm 5.45(n=238)$

$71.7(n=160)$

$28.3(n=63)$

$49.3(n=113)$

$34.5(n=79)$

$7.0(n=16)$

$7.0(n=16)$

$2.2(n=5)$

$27.9(n=62)$

$37.8(n=84)$

$22.5(n=50)$

$11.7(n=26)$

$90.2(n=202)$

$9.8(n=22)$

$2964 \pm 849$ ( $n=234)$ $38(36-40)(n=238)$ $75.6(n=233)$

$24.4(n=75)$

$0.08 \pm 0.98(n=246)$

$0.03 \pm 1.03(n=251)$

$0.10 \pm 1.02(n=251)$

$77.9(n=194)$

$22.1(n=55)$

$54.0(n=227)$

$46.0(n=193)$

$99.5(n=418)$

$0.5(n=2)$

$<0.001$

$98.8(n=397)$

$1.2(n=5)$

$24.55 \pm 4.91(n=383)$

0.01

$<0.001$

$28.91 \pm 4.74(n=420)$

0.02

$53.1(n=215)$

46.9 ( $n=190)$

$<0.001$

$44.8(n=186)$

$34.9(n=145)$

$6.7(n=28)$

$9.4(n=39)$

$4.1(n=17)$

$<0.001$

$24.3(n=98)$

$43.1(n=174)$

$19.3(n=78)$

$13.4(n=54)$

0.02

$85.0(n=357)$

$15.0(n=63)$

$40(38-40)(n=420)$

$3368 \pm 619(n=418)$

0.11

0.23

0.04

001

Data are presented as \%, mean \pm SD or median (interquartile range), unless otherwise stated. FEV1: forced expiratory volume in 1 s; FVC: forced vital capacity; FEF25-75\%: forced expiratory flow at $25-75 \%$ of FVC; BMI: body mass index. " : lung function SD Scores are standardised by age, sex and height; ": data are presented as median (interquartile range), as this variable has a skewed distribution.

Table 5 and figure 3 show the associations between HDP and childhood lung function. After controlling for potential confounders (model 3), there was weak evidence to suggest that compared to children of normotensive mothers, FEV1 and FEF25-75\% were lower in children whose mothers had had pre-eclampsia.

In order to explore potential mediation of associations by fetal growth retardation and prematurity, we controlled additionally for birthweight and gestational age (online supplementary table E2). This attenuated 


\begin{tabular}{|c|c|c|c|}
\hline & Model 1 & Model 2 & Model 3 \\
\hline \multicolumn{4}{|l|}{ Wheezing at age 18 months ${ }^{\#}$} \\
\hline Normotensive (reference) & 1 & 1 & 1 \\
\hline Gestational hypertension & $0.89(0.78-1.02)$ & $0.90(0.78-1.04)$ & $0.92(0.79-1.06)$ \\
\hline Pre-eclampsia & $1.16(0.84-1.60)$ & $1.27(0.91-1.76)$ & $1.31(0.94-1.82)$ \\
\hline Hypertension before pregnancy & $1.06(0.84-1.35)$ & $1.07(0.84-1.37)$ & $1.08(0.84-1.38)$ \\
\hline \multicolumn{4}{|l|}{ Wheezing at age 7 years $\pi$} \\
\hline Normotensive (reference) & 1 & 1 & 1 \\
\hline Gestational hypertension & $1.10(0.89-1.37)$ & $1.08(0.87-1.35)$ & $1.09(0.87-1.37)$ \\
\hline Pre-eclampsia & $0.96(0.54-1.71)$ & $0.98(0.54-1.76)$ & $0.98(0.55-1.77)$ \\
\hline Hypertension before pregnancy & $1.69(1.21-2.36)$ & $1.62(1.15-2.28)$ & $1.63(1.16-2.31)$ \\
\hline
\end{tabular}

Data are presented as OR (95\% CI). Model 1: adjusted for offspring sex; model 2: additionally adjusted for maternal asthma, maternal pre-pregnancy body mass index, maternal age, maternal parity, maternal education, multiple pregnancy and maternal ethnicity; model 3: additionally adjusted for maternal smoking during pregnancy. ${ }^{\#}: \mathrm{n}=9076 ;{ }^{\text {ๆ }}$ : $\mathrm{n}=7026$.

the effect estimates for the relations between pre-eclampsia and early wheezing, FEV1 and FEF25-75\%; however, the associations between hypertension before pregnancy and asthma and wheezing at 7 years were not attenuated.

\section{Discussion}

In this population-based birth cohort study we have found weak evidence for an association between pre-eclampsia and wheezing at 18 months and impaired ventilatory function later in childhood. Our findings for pre-eclampsia and early wheezing are in keeping with recent results from a large meta-analysis [4]. In that study, effect estimates were stronger for recurrent wheezing than for ever-wheezing. We must await new data from other large studies to see whether our findings for lung function are robustly replicated; to date, only one, relatively small study reported no association between pre-eclampsia and childhood lung function [3]. We also found that hypertension before pregnancy, but not gestational hypertension, was positively associated with wheezing and asthma at 7 years in the offspring. To our

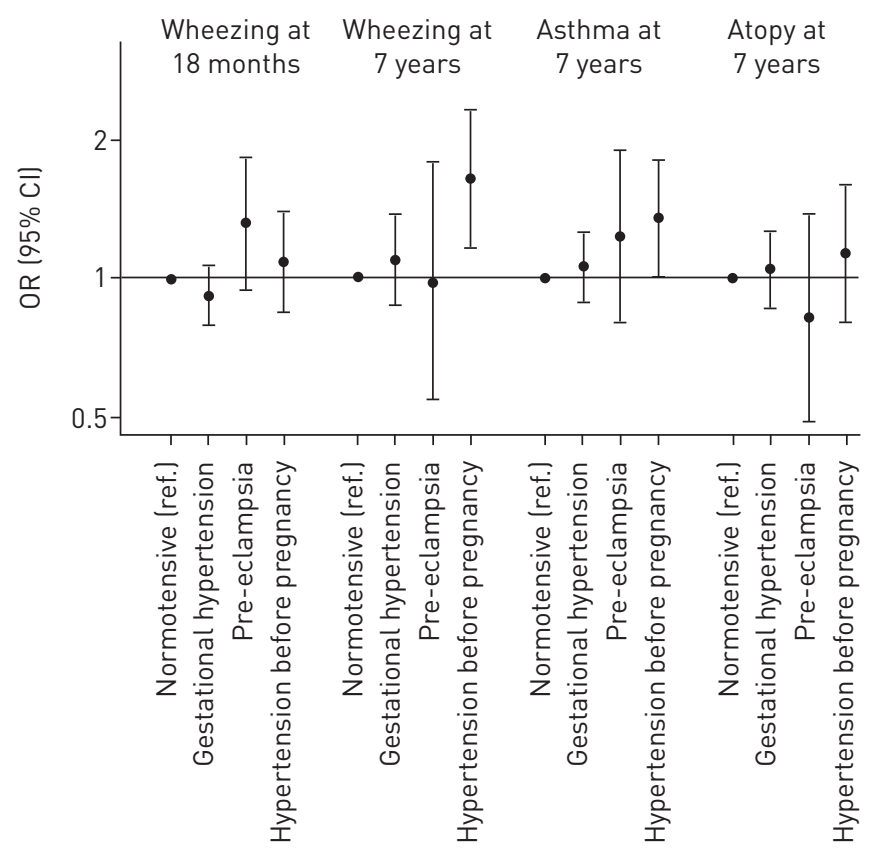

FIGURE 2 Odds ratios (95\% Cl) for early childhood wheezing and later childhood wheezing, asthma and atopy associated with maternal hypertensive disorders of pregnancy in the confounder-adjusted model ladjusted for offspring sex, maternal asthma, maternal pre-pregnancy body mass index, maternal age, maternal parity, maternal education, multiple pregnancy, maternal ethnicity and maternal smoking during pregnancy (model 3)). 
TABLE 3 Odds ratios for asthma at age 7 years associated with maternal hypertensive disorders of pregnancy

\begin{tabular}{lccc} 
& Model 1 & Model 2 & Model 3 \\
\hline Normotensive (reference) & 1 & 1 & 1 \\
Gestational hypertension & $1.08(0.91-1.28)$ & $1.04(0.87-1.24)$ & $1.05(0.88-1.25)$ \\
Pre-eclampsia & $1.23(0.81-1.86)$ & $1.20(0.78-1.83)$ & $1.23(0.80-1.88)$ \\
Hypertension before pregnancy & $1.39(1.05-1.85)$ & $1.33(1.00-1.78)$ & $1.34(1.00-1.79)$
\end{tabular}

Data are presented as OR $(95 \% \mathrm{CI}) . \mathrm{n}=6974$. Model 1: adjusted for offspring sex; model 2: additionally adjusted for maternal asthma, maternal pre-pregnancy body mass index, maternal age, maternal parity, maternal education, multiple pregnancy and maternal ethnicity; model 3: additionally adjusted for maternal smoking during pregnancy.

knowledge this has not been reported previously and needs replicating. We know of no previous studies that have considered the specific association of maternal hypertension before pregnancy with offspring wheezing and asthma. A previous study of maternal hypertension (including all forms of HDP) and childhood asthma was negative [8], and results of two studies of the relationship between maternal hypertension and pre-eclampsia (analysed in combination) and childhood wheezing and asthma were conflicting $[5,6]$. However, in these studies gestational hypertension and hypertension before pregnancy were not distinguished and analysed separately. In our study, pre-eclampsia was not associated with childhood asthma, which is in keeping with two previous studies [3,7], although, given the confidence limits around the effect estimates in our study, we cannot rule out a positive association; despite ALSPAC's size, the number of females with pre-eclampsia was still small, and therefore confidence intervals were wide.

We did not find an association between pre-eclampsia and atopy. While one small study reported a positive association between pre-eclampsia or placental abruption and severe atopy in the adolescent offspring, the study was limited to deliveries by Caesarean section, and pre-eclampsia and placental abruption were not analysed separately [11]. The only other study to examine the relationship between pre-eclampsia and atopy reported a positive association between severe pre-eclampsia and severe atopy only [3]. We did not attempt to sub-analyse our data further with respect to severity of pre-eclampsia or atopy because statistical power would have been compromised. The lack of a positive association between pre-eclampsia and atopy in our study suggests that pre-eclampsia is unlikely to explain any of the well-established relationships between parity (birth order) and atopy in the offspring, even though pre-eclampsia is more common in first pregnancies and atopy is more common in firstborns [22, 23].

\section{Potential mechanisms}

As pre-eclampsia is associated with low birthweight and prematurity [24, 25], and given that low birthweight and prematurity have been associated independently with wheezing, asthma and impaired lung function in children [26-30], it is plausible that a relationship between pre-eclampsia and adverse childhood respiratory outcomes, if causal, may be mediated through detrimental effects on fetal growth or gestational age at birth. In support of this hypothesis, and making key assumptions of mediation analysis [20, 21], we found that controlling for birthweight and gestational age substantially attenuated associations between pre-eclampsia and early childhood wheezing and lung function. This has not been explored in previous studies, with the

\begin{tabular}{|c|c|c|c|}
\hline & Model 1 & Model 2 & Model 3 \\
\hline Normotensive (reference) & 1 & 1 & 1 \\
\hline Gestational hypertension & $1.04(0.86-1.25)$ & $1.05(0.87-1.28)$ & $1.04(0.86-1.26)$ \\
\hline Pre-eclampsia & $0.81(0.49-1.33)$ & $0.83(0.50-1.39)$ & $0.82(0.49-1.37)$ \\
\hline Hypertension before pregnancy & $1.14(0.82-1.58)$ & $1.13(0.81-1.58)$ & $1.13(0.81-1.58)$ \\
\hline
\end{tabular}

Data are presented as $\mathrm{OR}(95 \% \mathrm{CI}) . \mathrm{n}=5532$. Model 1: adjusted for offspring sex; model 2: additionally adjusted for maternal asthma, maternal pre-pregnancy body mass index, maternal age, maternal parity, maternal education, multiple pregnancy and maternal ethnicity; model 3: additionally adjusted for maternal smoking during pregnancy. 


\begin{tabular}{|c|c|c|c|}
\hline & Model 1 & Model 2 & Model 3 \\
\hline \multicolumn{4}{|l|}{ FEV 1} \\
\hline Normotensive (reference) & 0 & 0 & 0 \\
\hline Gestational hypertension & $0.04(-0.03-0.12)$ & $0.04(-0.04-0.12)$ & $0.04(-0.04-0.12)$ \\
\hline Pre-eclampsia & $-0.14(-0.33-0.05)$ & $-0.13(-0.32-0.06)$ & $-0.14(-0.33-0.06)$ \\
\hline Hypertension before pregnancy & $0.09(-0.05-0.23)$ & $0.10(-0.04-0.23)$ & $0.09(-0.04-0.23)$ \\
\hline \multicolumn{4}{|l|}{ FVC } \\
\hline Normotensive (reference) & 0 & 0 & 0 \\
\hline Gestational hypertension & $0.06(-0.02-0.13)$ & $0.05(-0.02-0.13)$ & $0.06(-0.02-0.13)$ \\
\hline Pre-eclampsia & $-0.07(-0.26-0.12)$ & $-0.08(-0.27-0.11)$ & $-0.07(-0.26-0.12)$ \\
\hline Hypertension before pregnancy & $0.04(-0.09-0.18)$ & $0.04(-0.09-0.18)$ & $0.04(-0.09-0.18)$ \\
\hline \multicolumn{4}{|l|}{ FEF $25-75 \%$} \\
\hline Normotensive (reference) & 0 & 0 & 0 \\
\hline Gestational hypertension & $-0.02(-0.10-0.05)$ & $-0.01(-0.09-0.06)$ & $-0.02(-0.10-0.05)$ \\
\hline Pre-eclampsia & $-0.15(-0.34-0.04)$ & $-0.13(-0.32-0.06)$ & $-0.15(-0.34-0.04)$ \\
\hline Hypertension before pregnancy & $0.08(-0.05-0.22)$ & $0.09(-0.05-0.22)$ & $0.08(-0.05-0.22)$ \\
\hline
\end{tabular}

Data are presented as mean difference SD Scores (95\% CI). SD Scores are adjusted for age, sex and height. $\mathrm{n}=5601$. Model 1: adjusted for offspring sex; model 2: additionally adjusted for maternal asthma, maternal pre-pregnancy body mass index, maternal age, maternal parity, maternal education, multiple pregnancy and maternal ethnicity; model 3: additionally adjusted for maternal smoking during pregnancy. FEV1: forced expiratory volume in $1 \mathrm{~s}$; FVC: forced vital capacity; FEF25-75\%: forced expiratory flow at $25-75 \%$ of FVC.

exception of a recent meta-analysis, which confirmed that such adjustment led to moderate change in the risk estimate for the relation between pre-eclampsia and early childhood wheezing, although the magnitude of change was not quantified [4]. In our study, deficits in lung function associated with pre-eclampsia were stronger for $\mathrm{FEV}_{1}$ and $\mathrm{FEF}_{25}$-75\% than for FVC, suggesting detrimental effects on fetal airway growth. We speculate that the mechanism underlying such effects may involve overproduction of anti-angiogenic factors

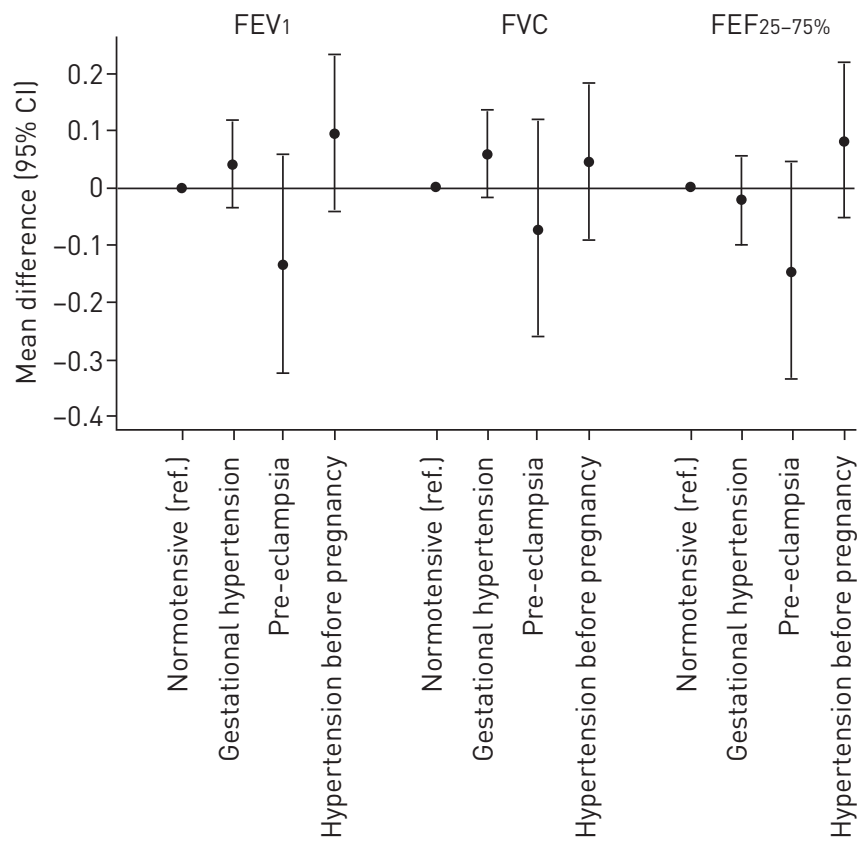

FIGURE 3 Mean differences $(95 \% \mathrm{CI})$ in lung function outcome SD scores at age 8 years associated with maternal hypertensive disorders of pregnancy in the confounder-adjusted model ladjusted for offspring sex, maternal asthma, maternal pre-pregnancy body mass index, maternal age, maternal parity, maternal education, multiple pregnancy, maternal ethnicity and maternal smoking during pregnancy (model 3)). Lung function SD scores are standardised by age, sex and height. FEV1: forced expiratory volume in 1 s; FVC: forced vital capacity; FEF25-75\%: forced expiratory flow at $25-75 \%$ of FVC. 
in amniotic fluid, which disrupts lung growth [31]; animal work has suggested that a similar mechanism may explain the link between pre-eclampsia and bronchopulmonary dysplasia in humans [32]. We have previously shown that pre-eclampsia is related to babies being small for gestational age, while gestational hypertension is not in this cohort [25]; this might explain why pre-eclampsia, but not gestational hypertension, was associated with early wheezing and reduced lung function. In contrast, the relationships between hypertension before pregnancy and childhood wheezing and asthma were not attenuated by controlling for birthweight and gestational age; this suggests that, if these associations are causal, they are unlikely to be mediated through impaired fetal growth or prematurity. Nor are they likely to be explained by atopy, given that hypertension before pregnancy was unrelated to this outcome. We cannot offer an alternative mechanistic explanation for the associations with wheezing and asthma. A lack of a positive association between pre-eclampsia and atopy is perhaps unsurprising, given that pre-eclampsia is associated with a T-helper cell 1 predominant immune profile [33].

\section{Strengths and limitations}

Aside from ALSPAC's size and population-based prospective design, a strength of our study is that we were able to define gestational hypertension and pre-eclampsia using data abstracted from the mothers' obstetric records, applying a standard definition of HDP to the blood pressure and proteinuria measurements themselves, rather than reliance on self-report. We were also able to distinguish the three conditions of hypertension before pregnancy, gestational hypertension and pre-eclampsia, in contrast with some previous studies [5, 6]; grouping the three hypertensive disorders for analysis may potentially dilute associations. However, despite the size of ALSPAC, we lacked power to exclude a possible modest detrimental effect of pre-eclampsia on early wheezing and later lung function, because pre-eclampsia is not a common condition; more definitive results would require investigation in larger birth cohorts or through meta-analysis of multiple cohorts. While we cannot rule out the possibility that the associations between hypertension before pregnancy and childhood wheezing/asthma, and the weak associations between pre-eclampsia and early wheezing and impaired lung function, might be explained by uncontrolled or residual confounding, we controlled for a large number of potential confounders. These included maternal smoking in pregnancy, which is associated with a lower incidence of pre-eclampsia [19] and would therefore negatively confound the associations between pre-eclampsia and respiratory outcomes, such that adjustment for maternal smoking would strengthen, not weaken, these associations; better measurement of maternal smoking would therefore be expected to increase the magnitude of associations with lung function further as it is likely that maternal smoking is underreported. Another important potential confounder that we considered was maternal asthma, which not only increases the risk of asthma in the offspring, but is also associated with a higher incidence of HDP (and low birthweight and prematurity) [34-36].

We acknowledge that there may have been some misclassification of maternally-reported outcomes, especially early childhood wheezing. Similarly, we cannot rule out the possibility of misclassification of the HDP, especially hypertension before pregnancy, which relied on maternal self-report. However, as misclassification of outcome is likely to be nondifferential with respect to the exposures of interest (and vice versa), this would be expected to bias effect estimates towards the null; in other words, the magnitude of associations would be underestimated. Furthermore, given that information on gestational hypertension and pre-eclampsia was obtained from obstetric records, we believe that any misclassification of these exposures will be less than in previous studies which have relied on maternal self-report of these disorders. As with any longitudinal study, data were not complete on exposures, outcomes and confounders for the whole cohort. Therefore, we cannot rule out the possibility that exclusion of children without complete information might have biased our findings. However, we can see no reason why associations between HDP and the outcomes of interest would be different in those included and those excluded.

\section{Conclusion}

We conclude that gestational hypertension is unlikely to increase the risk of asthma, atopy or impaired lung function in the offspring, which is reassuring given that this disorder is not uncommon. Hypertension before pregnancy may be a risk factor for childhood wheezing and asthma, although this observation requires replication. Determining whether pre-eclampsia is associated with impaired childhood lung function will require larger studies which are able to analyse pre-eclampsia separately from gestational hypertension and hypertension before pregnancy and adjust for a range of potential confounders.

\section{Acknowledgements}

We are extremely grateful to all the families who took part in this study, the midwives for their help in recruiting them, and the whole ALSPAC team, which includes interviewers, computer and laboratory technicians, clerical workers, research scientists, volunteers, managers, receptionists and nurses. 


\section{References}

1 Henderson AJ, Warner JO. Fetal origins of asthma. Semin Fetal Neonatal Med 2012; 17: 82-91.

2 Stick SM, Burton PR, Gurrin L, et al. Effects of maternal smoking during pregnancy and a family history of asthma on respiratory function in newborn infants. Lancet 1996; 348: 1060-1064.

3 Byberg K, Ogland B, Eide G, et al. Birth after preeclamptic pregnancies: association with allergic sensitization and allergic rhinoconjunctivitis in late childhood; a historically matched cohort study. BMC Pediatr 2014; $14: 101$.

4 Zugna D, Galassi C, Annesi-Maesano I, et al. Maternal complications in pregnancy and wheezing in early childhood: a pooled analysis of 14 birth cohorts. Int J Epidemiol 2015; 44: 199-208.

5 Rusconi F, Galassi C, Forastiere F, et al. Maternal complications and procedures in pregnancy and at birth and wheezing phenotypes in children. Am J Respir Crit Care Med 2007; 175: 16-21.

6 Nafstad P, Magnus P, Jaakkola JJ. Risk of childhood asthma and allergic rhinitis in relation to pregnancy complications. J Allergy Clin Immunol 2000; 106: 867-873.

7 Nafstad P, Samuelsen SO, Irgens LM, et al. Pregnancy complications and the risk of asthma among Norwegians born between 1967 and 1993. Eur J Epidemiol 2003; 18: 755-761.

8 Annesi-Maesano I, Moreau D, Strachan D. In utero and perinatal complications preceding asthma. Allergy 2001; 56: 491-497.

9 Roberts CL, Ford JB, Algert CS, et al. Population-based trends in pregnancy hypertension and pre-eclampsia: an international comparative study. BMJ Open 2011; 1: e000101.

10 Visintin C, Mugglestone MA, Almerie MQ, et al. Management of hypertensive disorders during pregnancy: summary of NICE guidance. BMJ 2010; 341: c2207.

11 Keski-Nisula L, Heinonen S, Remes S, et al. Pre-eclampsia, placental abruption and increased risk of atopic sensitization in male adolescent offspring. Am J Reprod Immunol 2009; 62: 293-300.

12 Boyd A, Golding J, Macleod J, et al. Cohort profile: the 'children of the 90s' - the index offspring of the Avon Longitudinal Study of Parents and Children. Int J Epidemiol 2013; 42: 111-127.

13 Fraser A, Macdonald-Wallis C, Tilling K, et al. Cohort profile: the Avon Longitudinal Study of Parents and Children: ALSPAC mothers cohort. Int J Epidemiol 2013; 42: 97-110.

14 Brown MA, Lindheimer MD, de Swiet M, et al. The classification and diagnosis of the hypertensive disorders of pregnancy: statement from the International Society for the Study of Hypertension in Pregnancy (ISSHP). Hypertens Pregnancy 2001; 20: ix-xiv.

15 Chinn S, Rona RJ. Height and age adjustment for cross sectional studies of lung function in children aged 6-11 years. Thorax 1992; 47: 707-714.

16 American Thoracic Society. Standardization of spirometry, 1994 update. Am J Respir Crit Care Med 1995; 152: 1107-1136.

17 Arets HGM, Brackel HJL, van der Ent CK. Forced expiratory manoeuvres in children: do they meet ATS and ERS criteria for spirometry? Eur Respir J 2001; 18: 655-660.

18 Roberts G, Peckitt C, Northstone K, et al. Relationship between aeroallergen and food allergen sensitization in childhood. Clin Exp Allergy 2005; 35: 933-940.

19 England L, Zhang J. Smoking and risk of preeclampsia: a systematic review. Front Biosci 2007; 12: 2471-2483.

20 Robins JM, Greenland S. Identifiability and exchangeability for direct and indirect effects. Epidemiology 1992; 3: $143-155$.

21 Cole SR, Hernán MA. Fallibility in estimating direct effects. Int J Epidemiol 2002; 31: 163-165.

22 Duckitt K, Harrington D. Risk factors for pre-eclampsia at antenatal booking: systematic review of controlled studies. BMJ 2005; 330: 565

23 Strachan DP. Hay fever, hygiene, and household size. BMJ 1989; 299: 1259-1260.

24 Xiong X, Mayes D, Demianczuk N, et al. Impact of pregnancy-induced hypertension on fetal growth. Am J Obstet Gynecol 1999; 180: 207-213.

25 Geelhoed JJ, Fraser A, Tilling K, et al. Preeclampsia and gestational hypertension are associated with childhood blood pressure independently of family adiposity measures: The Avon Longitudinal Study of Parents and Children. Circulation 2010; 122: 1192-1199.

26 Rona RJ, Gulliford MC, Chinn S. Effects of prematurity and intrauterine growth on respiratory health and lung function in childhood. BMJ 1993; 306: 817-820.

27 Jaakkola JJK, Ahmed P, Ieromnimon A, et al. Preterm delivery and asthma: a systematic review and meta-analysis. J Allergy Clin Immunol 2006; 118: 823-830.

28 Kotecha SJ, Watkins WJ, Heron J, et al. Spirometric lung function in school-age children: effect of intrauterine growth retardation and catch-up growth. Am J Respir Crit Care Med 2010; 181: 969-974.

29 Kotecha SJ, Watkins WJ, Paranjothy S, et al. Effect of late preterm birth on longitudinal lung spirometry in school age children and adolescents. Thorax 2012; 67: 54-61.

30 Sonnenschein-van der Voort A, Arends LR, de Jongste JC, et al. Preterm birth, infant weight gain, and childhood asthma risk: a meta-analysis of 147,000 European children. J Allergy Clin Immunol 2014; 133: 1317-1329.

31 Tang JR, Karumanchi SA, Seedorf G, et al. Excess soluble vascular endothelial growth factor receptor-1 in amniotic fluid impairs lung growth in rats: linking preeclampsia with bronchopulmonary dysplasia. Am J Physiol Lung Cell Mol Physiol 2012; 302: L36-L46.

32 Hansen AR, Barnés CM, Folkman J, et al. Maternal preeclampsia predicts the development of bronchopulmonary dysplasia. J Pediatr 2010; 156: 532-536.

33 Saito S, Sakai M. Th1/Th2 balance in preeclampsia. J Reprod Immunol 2003; 59: 161-173.

34 Liu S, Wen SW, Demissie K, et al. Maternal asthma and pregnancy outcomes: a retrospective cohort study. Am J Obstet Gynecol 2001; 184: 90-96.

35 Demissie K, Breckenridge MB, Rhoads GG. Infant and maternal outcomes in the pregnancies of asthmatic women. Am J Respir Crit Care Med 1998; 158: 1091-1095.

36 Enriquez R, Griffin MR, Carroll KN, et al. Effect of maternal asthma and asthma control on pregnancy and perinatal outcomes. J Allergy Clin Immunol 2007; 120: 625-630. 